\title{
Distributed non-cooperative MPC with neighbor-to-neighbor communication *
}

\author{
Marcello Farina, Riccardo Scattolini \\ Dipartimento di Elettronica e Informazione, Politecnico di Milano, Milan, \\ Italy
}

\begin{abstract}
This paper presents a novel distributed Model Predictive Control (MPC) algorithm. This method enjoys the following properties: (i) input and state constraints can be considered; (ii) under mild assumptions, convergence of the closed loop control system is proved; (iii) it is not necessary for each subsystem to know the dynamical models governing the trajectories of the other subsystems; (iv) the transmission of information is limited, in that each subsystem only needs the reference trajectories of the state variables of its neighbors. A simulation example is reported to illustrate the main characteristics and performance of the algorithm.
\end{abstract}

Keywords: Distributed control, large scale systems, model predictive control, plant-wide control.

\section{INTRODUCTION}

Many large scale and spatially distributed systems, such as power networks, transport networks and hydro power plants (Negenborn et al., 2009; Šiljac, 1978), motivate the development of distributed control structures with limited local computation and transmission requirements. In the context of Model Predictive Control (MPC), many distributed control algorithms have been proposed. Specifically, completely decentralized architectures (Raimondo et al., 2006; Barcelli and Bemporad, 2009), distributed schemes (see, e.g., Dunbar (2007); Liu et al. (2010, 2009); Venkat et al. (2008); Stewart et al. (2010)) and coordinated control techniques for dynamically decoupled systems (Dunbar and Murray, 2006; Ferrari-Trecate et al., 2009; Richards and How, 2007; Trodden and Richards, 2010) have been proposed.

According to the taxonomy proposed in (Scattolini, 2009), distributed MPC techniques can be classified according to the topology of the transmission network (i.e., fully connected or partially connected networks), to the information exchange protocol needed (i.e., non-iterative or iterative algorithms) and to the type of cost function which is optimized (i.e., cooperative or non-cooperative algorithms). In Dunbar (2007) a non-iterative, non-cooperative distributed MPC technique is proposed for continuous-time systems based on neighbor-toneighbor information exchange. In Liu et al. $(2009,2010)$ a non-iterative sequential (partially connected) algorithm and a novel iterative fully connected one are proposed. In (Venkat et al., 2008; Stewart et al., 2010) a cooperative fully connected output-feedback MPC algorithm for discrete time systems is discussed, where only input constraints can be assigned and full knowledge on the system dynamics is required to all the subsystems.

In this work we propose a non-iterative, non-cooperative MPC algorithm where a neighbor-to-neighbor (i.e., partially connected) communication network and partial (regional) struc-

\footnotetext{
* This research has been supported by the European 7th framework STREP project "Hierarchical and distributed model predictive control (HD-MPC)", contract number INFSO-ICT-223854.

e-mail:\{farina,scattolini $\} @$ elet.polimi.it
}

tural information are needed. The rationale of the proposed technique is that, at each sampling time, each subsystem sends to its neighbors information about its future reference trajectory and guarantees that the actual trajectory lies within a certain bound in the neighborhood of the reference one. Then, a robust MPC approach inspired by Mayne et al. (2005) provides a tool for the statement of the local optimization problems solved by each subsystem.

The highlights of the proposed approach are: $(i)$ input and state constraints can be considered and, under mild assumptions on the existence of a suitable decentralized auxiliary control law, convergence of the closed loop control system is proved; (ii) it is not necessary for each subsystem to know the dynamical models governing the trajectories of the other subsystems (not even the ones of the neighbors); (iii) the transmission of information is limited, in that each subsystem needs the reference trajectories only of the variables of one's neighbors which actually affect its dynamics; (iv) its rationale is very similar to the one of the MPC algorithms presently employed in industry, where reference trajectories tailored on the dynamics of the system under control are used.

Notation. We use the short-hand $\mathbf{v}=\left(v_{1}, \ldots, v_{s}\right)$ to denote a column vector with $s$ (not necessarily scalar) components $v_{1}$, $\ldots, v_{s}$. The symbol $\oplus$ denotes the Minkowski sum, namely $C=A \oplus B$ if and only if $C=\{c: c=a+b$, for all $a \in A, b \in B\}$. We also denote $\bigoplus_{i=1}^{M} A_{i}=A_{1} \oplus \cdots \oplus A_{M}$. For a discrete-time signal $s_{t}$ and $a, b \in \mathbb{N}, a \leq b$, we denote $\left(s_{a}, s_{a+1}, \ldots, s_{b}\right)$ with $s_{[a: b]}$. Finally, a continuous function $\alpha: \mathbb{R}_{+} \rightarrow \mathbb{R}_{+}$is a $\mathscr{K}_{\infty}$ function iff $\alpha(0)=0$, it is strictly increasing and $\alpha(s) \rightarrow+\infty$ as $s \rightarrow+\infty$.

\section{PARTITIONED SYSTEMS}

Consider a process which obeys to the linear dynamics

$$
\mathbf{x}_{t+1}=\mathbf{A} \mathbf{x}_{t}+\mathbf{B} \mathbf{u}_{t},
$$

where $\mathbf{x}_{t} \in \mathbb{R}^{n}$ is the state vector and $\mathbf{u}_{t} \in \mathbb{R}^{m}$ is the input signal. Let the system (1) be partitioned in $M$ low order interconnected non overlapping subsystems, where a generic submodel has $x_{t}^{[i]} \in \mathbb{R}^{n_{i}}$ as state vector, i.e., $\mathbf{x}_{t}=\left(x_{t}^{[1]}, \ldots, x_{t}^{[M]}\right)$ and $\sum_{i=1}^{M} n_{i}=n$. 
According to this decomposition, the state transition matrices $A_{11} \in \mathbb{R}^{n_{1} \times n_{1}}, \ldots, A_{M M} \in \mathbb{R}^{n_{M} \times n_{M}}$ of the $M$ subsystems are diagonal blocks of $\mathbf{A}$, whereas the non-diagonal blocks of $\mathbf{A}$ (i.e., $A_{i j}$, with $i \neq j$ ) define the coupling terms between subsystems. The partition performed on the system induces an interconnected network of subsystems, which can be naturally described by means of a directed graph $\mathscr{G}=(\mathscr{V}, \mathscr{H})$, where the nodes in $\mathscr{V}$ are the subsystems and the edge $(j, i)$ in the set $\mathscr{H} \subseteq \mathscr{V} \times \mathscr{V}$ models that the state of $j$ affects the dynamics of subsystem $i$. In particular, $(j, i) \in \mathscr{H}$ if and only if $A_{i j} \neq 0$. We denote as $\mathscr{N}_{i}$ the set of neighbors of subsystem $i$ (which excludes $i)$ i.e., $\mathscr{N}_{i}=\{j \mid j \neq i$ and $(j, i) \in \mathscr{H}\}$.

Furthermore, we assume that the input $\mathbf{u}_{t}$ can be partitioned into a set of $M$ input vectors $u_{t}^{[i]} \in \mathbb{R}^{m_{i}}$, with $i=1, \ldots, M$, where $u_{t}^{[i]}$ directly affects only the state of the $i$-th subsystem $x_{t}^{[i]}$. This implies that $\mathbf{B}$ has a block diagonal structure $\mathbf{B}=\operatorname{diag}\left(B_{1}, \ldots, B_{M}\right)$, where $B_{i} \in \mathbb{R}^{n_{i} \times m_{i}}$ for all $i=1, \ldots, M$. It finally results that the $i$-th subprocess obeys to the linear dynamics

$$
x_{t+1}^{[i]}=A_{i i} x_{t}^{[i]}+B_{i} u_{t}^{[i]}+\sum_{j \in \mathscr{N}_{i}} A_{i j} x_{t}^{[j]}
$$

where $x_{t}^{[i]} \in \mathbb{X}_{i} \subseteq \mathbb{R}^{n_{i}}$ is the state vector and $u_{t}^{[i]} \in \mathbb{U}_{i} \subseteq \mathbb{R}^{m_{i}}$ is the input vector. The sets $\mathbb{X}_{i}$ and $\mathbb{U}_{i}$ are convex neighborhoods of the origin. Furthermore we define $\mathbb{X}=\prod_{i=1}^{M} \mathbb{X}_{i} \subseteq \mathbb{R}^{n}$ and $\mathbb{U}=\prod_{i=1}^{M} \mathbb{U}_{i}$, which are convex by convexity of $\mathbb{X}_{i}$ and $\mathbb{U}_{i}$, respectively, for $i=1, \ldots, M$. When $\mathbb{X}=\mathbb{R}^{n}$ and $\mathbb{U}=\mathbb{R}^{m}$ we say that the system is unconstrained.

Our aim is to design, for each subsystem $i$, an algorithm for computing an input sequence $u_{t}^{[i]}$ based on the state $x_{t}^{[i]}$ and some information which is transmitted by its neighbors $\mathscr{N}_{i}$, which guarantees closed loop asymptotic convergence to the origin of the state of the large scale system (1), the minimization of a given local cost function and constraint satisfaction. Specifically, we assume that each subsystem has a reference trajectory $\tilde{x}_{t}^{[i]}$ which is transmitted to the subsystems which have $i$ as neighbor, and which is incrementally defined (as better specified in the following). We also assume that one can guarantee that, for all $t \geq 0$, the real local state trajectory $x_{t}^{[i]}$ lies in a specified time-invariant neighborhood of $\tilde{x}_{t}^{[i]}$ i.e, $x^{[i]}-\tilde{x}_{t}^{[i]} \in \mathscr{E}_{i}$, where $0 \in \mathscr{E}_{i}$.

Letting $w_{t}^{[i]}=\sum_{j \in \mathscr{N}_{i}} A_{i j}\left(x_{t}^{[j]}-\tilde{x}_{t}^{[j]}\right)$, the $i$-th system model (2) can be written as follows

$$
x_{t+1}^{[i]}=A_{i i} x_{t}^{[i]}+B_{i} u_{t}^{[i]}+\sum_{j \in \mathscr{N}_{i}} A_{i j} \tilde{x}_{t}^{[j]}+w_{t}^{[i]}
$$

where the term $w_{t}^{[i]} \in \mathbb{W}_{i}=\bigoplus_{j \in \mathscr{N}_{i}} A_{i j} \mathscr{E}_{j}$ represents a bounded disturbance affecting equation (3) and $\sum_{j \in \mathscr{N}_{i}} A_{i j} \tilde{x}_{t}^{[j]}$ can be considered as a known input. Provided that, for all $i=1, \ldots, M$, the constraint $x^{[i]}-\tilde{x}_{t}^{[i]} \in \mathscr{E}_{i}$ can be successfully imposed (see Section 6 for a short discussion on this point) for all $t \geq 0$, we can cast the problem of designing a distributed MPC control law as the problem of designing a robust control law for the subsystem (3), for all $i=1, \ldots, M$.

For the statement of the local MPC sub-problems (which will be denoted $i$-MPC problems) we rely on the robust MPC algorithm presented in Mayne et al. (2005) for constrained linear systems with bounded disturbances. The main advantages of this approach are that no burdensome min-max problem is required to be solved on-line, and that it naturally provides the reference trajectory $\tilde{x}_{t}^{[i]}$, which is one of the key points of the algorithm presented in this paper.

\section{THE DISTRIBUTED MPC ALGORITHM}

As a preliminary step to the statement of the local $i$-MPC problem, we define the $i$-th subsystem nominal model associated to equation (3) as

$$
\hat{x}_{t+1}^{[i]}=A_{i i} \hat{x}_{t}^{[i]}+B_{i} \hat{u}_{t}^{[i]}+\sum_{j \in \mathscr{N}_{i}} A_{i j} \tilde{x}_{t}^{[j]}
$$

The control law for the real $i$-th subsystem (3) will be assigned, for all $t \geq 0$, according to

$$
u_{t}^{[i]}=\hat{u}_{t}^{[i]}+K_{i}^{a u x}\left(x_{t}^{[i]}-\hat{x}_{t}^{[i]}\right)
$$

where $K_{i}^{a u x}$ is a suitable control gain. Letting $z_{t}^{[i]}=x_{t}^{[i]}-\hat{x}_{t}^{[i]}$ from (3) and (5) we obtain

$$
z_{t+1}^{[i]}=\left(A_{i i}+B_{i} K_{i}^{\text {aux }}\right) z_{t}^{[i]}+w_{t}^{[i]}
$$

where $w_{t}^{[i]} \in \mathbb{W}_{i}$. Since $\mathbb{W}_{i}$ is bounded, if $\left(A_{i i}+B_{i} K_{i}^{\text {aux }}\right)$ is Schur, then there exists a robust positively invariant (RPI) set $Z_{i}$ for (6) such that, for all $z_{t}^{[i]} \in Z_{i}$, then $z_{t+1}^{[i]} \in Z_{i}$. A method for computing polytopic, robust positively invariant, outer approximations of the minimal robust positively invariant set is proposed in Raković et al. (2005). From (6) it follows that, if $u_{k}^{[i]}$ is computed as in (5) for all $k \geq t$, then

$$
x_{t}^{[i]}-\hat{x}_{t}^{[i]} \in Z_{i}
$$

implies that $x_{k}^{[i]}-\hat{x}_{k}^{[i]} \in Z_{i}$ for all $k \geq t$.

Write $x_{t}^{[i]}-\tilde{x}_{t}^{[i]}=\left(x_{t}^{[i]}-\hat{x}_{t}^{[i]}\right)+\left(\hat{x}_{t}^{[i]}-\tilde{x}_{t}^{[i]}\right)$ and define the set $E_{i}$ for all $i=1, \ldots, M$ as a set containing the origin and satisfying $E_{i} \oplus Z_{i} \subseteq \mathscr{E}_{i}$. Since, in view of (7), $x_{k}^{[i]}-\hat{x}_{k}^{[i]} \in Z_{i}$ for all $k \geq t$, if we also satisfy the constraint

$$
\hat{x}_{k}^{[i]}-\tilde{x}_{k}^{[i]} \in E_{i}
$$

for all $k \geq t$, then $x_{k}^{[i]}-\tilde{x}_{k}^{[i]} \in \mathscr{E}_{i}$ for all $k \geq t$, as required.

Now we are in the position to state the local minimization problem for all subsystems at instant $t$. Given the future reference trajectory of $i$ and its neighbors $\tilde{x}_{k}^{[j]}, k=t, \ldots, t+N-1$, $j \in \mathscr{N}_{i} \cup\{i\}$, the $i$-MPC problem consists in the following

$$
\min _{\hat{x}_{t}^{[i]}, \hat{u}_{[t: t+N-1]}^{[i]}} V_{i}^{N}\left(\hat{x}_{t}^{[i]}, \hat{u}_{[t: t+N-1]}^{[i]}\right)
$$

subject to the dynamic and static constraints (4), (7), (8), to

$$
\begin{aligned}
& \hat{x}_{k}^{[i]} \in \hat{\mathbb{X}}_{i} \\
& \hat{u}_{k}^{[i]} \in \hat{\mathbb{U}}_{i}
\end{aligned}
$$

where $\hat{\mathbb{X}}_{i} \oplus Z_{i} \subseteq \mathbb{X}_{i}$ and $\hat{\mathbb{U}}_{i} \oplus K Z_{i} \subseteq \mathbb{U}_{i}$, and to the terminal constraint

$$
\hat{x}_{t+N}^{[i]} \in \hat{\mathbb{X}}_{i}^{F}
$$

where $\hat{\mathbb{X}}_{i}^{F}$ is the $i$-th nominal subsystem terminal set, whose properties will be specified in the following.

$$
\begin{aligned}
& \text { The cost function } V_{i}^{N}\left(\hat{x}_{t}^{[i]}, \hat{u}_{[t: t+N-1]}^{[i]}\right) \text { is } \\
& \qquad V_{i}^{N}\left(\hat{x}_{t}^{[i]}, \hat{u}_{[t: t+N-1]}^{[i]}\right)=\sum_{k=t}^{t+N-1} l_{i}\left(\hat{x}_{k}^{[i]}, \hat{u}_{k}^{[i]}\right)+V_{i}^{F}\left(\hat{x}_{t+N}^{[i]}\right)
\end{aligned}
$$

where $l_{i}: \mathbb{R}^{n_{i}} \times \mathbb{R}^{m_{i}} \rightarrow \mathbb{R}_{+}$is the stage cost and $V_{i}^{F}: \mathbb{R}^{n_{i}} \rightarrow \mathbb{R}_{+}$ is the final cost. From now on, we assume that $l_{i}$ is defined 
in such a way that $l_{i}(0,0)=0$ and that there exists, for all $i=1, \ldots, M$, a $\mathscr{K}_{\infty}$ function $\alpha$ and a matrix $R_{i}$ satisfying $\operatorname{rank}\left(\left[B_{i}^{T} R_{i}^{T}\right]^{T}\right)=m_{i}$ such that $l_{i}\left(x^{[i]}, u^{[i]}\right) \geq \alpha\left(\left\|\left(x^{[i]}, R_{i} u^{[i]}\right)\right\|\right)$ for all $x^{[i]} \in \mathbb{R}^{n_{i}}, u^{[i]} \in \mathbb{R}^{m_{i}}$. Note that this assumption can always be fulfilled by a proper choice of the weight $R_{i}$ in the stage cost. As in Mayne et al. (2005), minimization is performed with respect both to the nominal system initial state $\hat{x}_{t}^{[i]}$ and to the nominal input trajectory $\hat{u}_{[t: t+N-1]}^{[i]}$. Letting the pair $\hat{x}_{t / t}^{[i]}, \hat{u}_{[t: t+N-1] / t}^{[i]}$ be the solution to the $i$-MPC problem (9) at time $t$, we assign the input to the nominal system (4), at time $t$, as $\hat{u}_{t / t}^{[i]}$. According to (5), the input to the real system (2), at instant $t$, is

$$
u_{t}^{[i]}=\hat{u}_{t / t}^{[i]}+K_{i}^{a u x}\left(x_{t}^{[i]}-\hat{x}_{t / t}^{[i]}\right)
$$

Furthermore, let us define as $\hat{x}_{k / t}^{[i]}$ the trajectory stemming from $\hat{x}_{t / t}^{[i]}$ and $\hat{u}_{[t: t+N-1] / t}^{[i]}$, in view of equation (4). The value of the reference state variable $\tilde{x}_{t+N}^{[i]}$ is set to

$$
\tilde{x}_{t+N}^{[i]}=\hat{x}_{t+N / t}^{[i]}
$$

We stress that we do not define, at each instant $t$, a new reference trajectory $\tilde{x}_{k}^{[i]}, k=t+1, \ldots, t+N$, but we append the value $\tilde{x}_{t+N}^{[i]}$ to the reference trajectory which has been already defined for $k \leq t+N-1$. For this reason $\tilde{x}_{t+N}^{[j]}$, for all $j \in \mathscr{N}_{i}$, is the only information which node $i$ must receive by its neighbors at instant $t$. Therefore we can conclude that, despite the offline design phase is rather complex, requiring the definition of suitable sets and cost functions, the on-line computational and communication loads are considerably limited.

\section{CONVERGENCE RESULTS}

The following definitions and assumptions are needed to state the main result of the paper.

The set of admissible initial conditions $\mathbf{x}_{0}=\left(x_{0}^{[1]}, \ldots, x_{0}^{[M]}\right)$ and initial reference trajectories $\tilde{x}_{[0: N-1]}^{[j]}$, for all $j=1 \ldots, M$ are defined as follows.

Definition 1. Letting $\mathbf{x}=\left(x^{[1]}, \ldots, x^{[M]}\right)$, we denote the feasibility region $\mathbb{X}^{N}$ for all the $i$-MPC problems as the set

$$
\begin{aligned}
\mathbb{X}^{N}:= & \left\{\mathbf{x}: \text { if } x_{0}^{[i]}=x^{[i]} \text { for all } i=1, \ldots, M\right. \\
& \text { then } \exists\left(\tilde{x}_{[0: N-1]}^{[1]}, \ldots, \tilde{x}_{[0: N-1]}^{[M]}\right),\left(\hat{x}_{0 / 0}^{[1]}, \ldots, \hat{x}_{0 / 0}^{[M]}\right), \\
& \left(\hat{u}_{[0: N-1]}^{[1]}, \ldots, \hat{u}_{[0: N-1]}^{M]}\right) \text { such that }(2),(7),(8),
\end{aligned}
$$

(10)-(12) are satisfied for all $i=1, \ldots, M\}$

We also denote, for each $\mathbf{x} \in \mathbb{X}^{N}$, the region of feasible initial reference trajectories as

$$
\begin{aligned}
\tilde{\mathbb{X}}_{\mathbf{x}}:= & \left\{\left(\tilde{x}_{[0: N-1]}^{[1]}, \ldots, \tilde{x}_{[0: N-1]}^{[M]}\right): \text { if } x_{0}^{[i]}=x^{[i]} \text { for all } i=1, \ldots, M\right. \\
& \text { then } \exists\left(\hat{x}_{0 / 0}^{11]}, \ldots, \hat{x}_{0 / 0}^{[M]}\right),\left(\hat{u}_{[0: N-1]}^{[1]}, \ldots, \hat{u}_{[0: N-1]}^{[M]}\right) \text { such that } \\
& (2),(7),(8),(10)-(12) \text { are satisfied for all } i=1, \ldots, M\}
\end{aligned}
$$

Assumption 1. The matrix $A_{i i}+B_{i} K_{i}^{\text {aux }}$ is Schur, for all $i=$ $1, \ldots, M$.

Assumption 2. Letting $\mathbf{K}^{\text {aux }}=\operatorname{diag}\left(K_{1}^{\text {aux }}, \ldots, K_{M}^{\text {aux }}\right), \hat{\mathbb{X}}=\prod_{i=1}^{M} \hat{\mathbb{X}}_{i}$, $\hat{\mathbb{U}}=\prod_{i=1}^{M} \hat{\mathbb{U}}_{i}$ and $\hat{\mathbb{X}}^{F}=\prod_{i=1}^{M} \hat{\mathbb{X}}_{i}^{F}$, it holds that:

(i) The matrix $\mathbf{A}+\mathbf{B K}^{\text {aux }}$ is Schur;

(ii) $\hat{\mathbb{X}}^{F} \subseteq \hat{\mathbb{X}}$ is an invariant set for $\hat{\mathbf{x}}^{+}=\left(\mathbf{A}+\mathbf{B} \mathbf{K}^{a u x}\right) \hat{\mathbf{x}}$;

(iii) $\hat{\mathbf{u}}=\mathbf{K}^{\text {aux }} \hat{\mathbf{x}} \in \hat{\mathbb{U}}$ for any $\hat{\mathbf{x}} \in \hat{\mathbb{X}}^{F}$; (iv) for all $\hat{\mathbf{x}} \in \hat{\mathbb{X}}^{F}$ and, for a given constant $\kappa>0$

$$
\mathbf{V}^{F}\left(\hat{\mathbf{x}}^{+}\right)-\mathbf{V}^{F}(\hat{\mathbf{x}}) \leq-(1+\kappa) \mathbf{l}(\hat{\mathbf{x}}, \hat{\mathbf{u}})
$$

where $\mathbf{V}^{F}(\hat{\mathbf{x}})=\sum_{i=1}^{M} V_{i}^{F}\left(\hat{x}^{[i]}\right)$ and $\mathbf{l}(\hat{\mathbf{x}}, \hat{\mathbf{u}})=\sum_{i=1}^{M} l_{i}\left(\hat{x}^{[i]}, \hat{u}^{[i]}\right)$. Assumption 3. Given the sets $\mathscr{E}_{i}$ and the RPI sets $Z_{i}$ for equations (6), there exists a real positive constant $\bar{\rho}_{E}>0$ such that $Z_{i} \oplus \mathscr{B}_{\bar{\rho}_{E}}(0) \subseteq \mathscr{E}_{i}$ for all $i=1, \ldots, M$, where $\mathscr{B}_{\bar{\rho}_{E}}(0)$ is a ball of radius $\bar{\rho}_{E}>0$ centered at the origin.

Now we are in the position to state the main result.

Theorem 1. Let Assumptions 1-3 be satisfied and let $E_{i}$ be a neighborhood of the origin satisfying $E_{i} \oplus Z_{i} \subseteq \mathscr{E}_{i}$. Then the trajectory $\mathbf{x}_{t}$, starting from any initial condition $\mathbf{x}_{0} \in \mathbb{X}^{N}$, asymptotically converges to the origin, provided that the initial reference trajectories are in $\tilde{\mathbb{X}}_{\mathbf{x}_{0}}$.

Proof. See Appendix A

\section{EXAMPLE}

Consider a fourth-order system with two input variables. The dynamics of the system is described by (4), where

$$
\mathbf{A}=\left[\begin{array}{ll}
A_{11} & A_{12} \\
A_{21} & A_{22}
\end{array}\right], \mathbf{B}=\left[\begin{array}{cc}
B_{1} & 0 \\
0 & B_{2}
\end{array}\right]
$$

and

$A_{11}=A_{22}=\left[\begin{array}{cc}0 & 1 \\ -1 & 2\end{array}\right], A_{12}=-A_{21}=\left[\begin{array}{cc}0.1 & 0.1 \\ 0 & 0.3\end{array}\right], B_{1}=B_{2}=\left[\begin{array}{l}0 \\ 1\end{array}\right]$

The following constraints are set to the input signals: $\left|u_{k}^{[1]}\right| \leq$ 2.5 and $\left|u_{k}^{[2]}\right| \leq 4$. We define $l_{i}\left(\hat{x}_{k}^{[i]}, \hat{u}_{k}^{[i]}\right)=\frac{1}{2}\left\|\hat{x}_{k}^{[i]}\right\|_{Q_{i}}^{2}$ and $V_{i}^{F}\left(\hat{x}_{t+N}^{[i]}\right)=\frac{1}{2}\left\|\hat{x}_{t+N}^{i]}\right\|_{P_{i}}^{2}$, where $P_{1}=P_{2}=\operatorname{diag}(1,3)$ and $Q_{1}=$ $Q_{2}=\operatorname{diag}(0.4593,0.4593)$. Setting $K_{1}=K_{2}=\left[\begin{array}{ll}1 & -2\end{array}\right]$, Assumptions 1 and 2 are verified. In the simulations, we set $N=4$ and the reference trajectories are initialized by simulating the subsystems controlled using the auxiliary control law, where the coupling terms are neglected, that is $\tilde{x}_{k}^{[i]}=\left(A_{i i}+B_{i} K_{i}\right)^{k} x_{0}^{[i]}$, for $k=0, \ldots, t+N-1$. A choice of the sets $Z_{i}, E_{i}$ and $\mathbb{W}_{i}$,
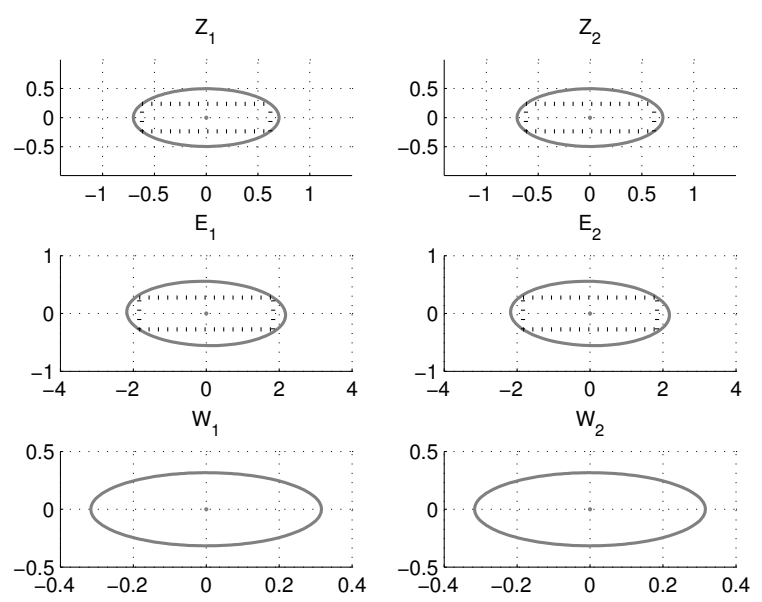

Fig. 1. Sets $Z_{i}, E_{i}$ and $\mathbb{W}_{i}, i=1,2$, chosen in the given example. The black dotted lines represent the real constraints exerted in the example.

$i=1,2$, consistent with Assumption 3, is shown in Fig. 1 (grey ellipsoids), where the black dotted lines represent the real constraints considered, for simplicity, in the solution of the 
constrained optimization problems $i$-MPC, $i=1,2$.

In Fig. 2 the plots of the optimal input trajectories obtained
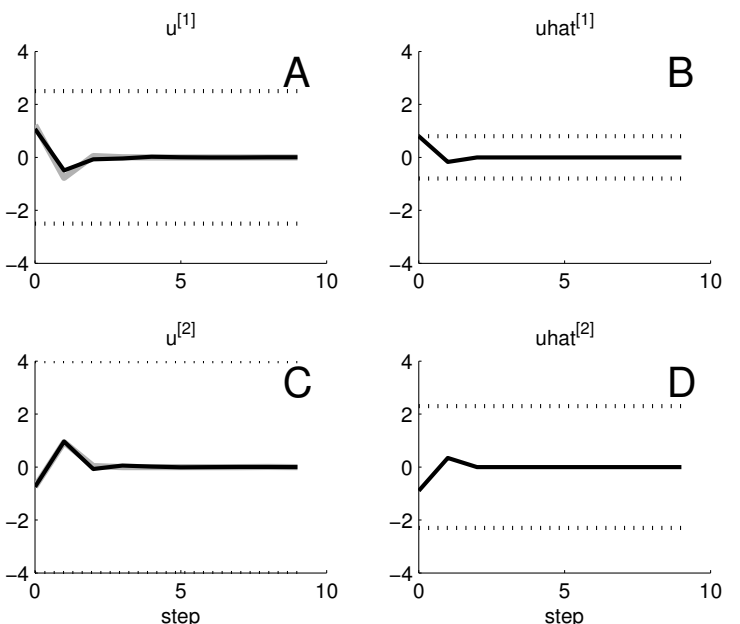

Fig. 2. Input trajectories. A: $u_{t}^{[1]}$ (black solid line), input obtained with cMPC (grey solid line), thresholds for $u_{t}^{[1]}$ (black dotted lines). B: $\hat{u}_{t}^{[1]}$ (black solid line), thresholds for $\hat{u}_{t / t}^{[1]}$ (black dotted lines). C: $u_{t}^{[2]}$ (black solid line), inputs obtained with cMPC (grey solid line), thresholds for $u_{t}^{[2]}$ (black dotted lines). D: $\hat{u}_{t / t}^{[2]}$ (black solid line), thresholds for $\hat{u}_{t}^{[2]}$ (black dotted lines).

with the distributed MPC algorithm (dMPC) are shown and compared with those obtained with a centralized MPC (cMPC). Notably, at time $t=0$ the constraint on $\hat{u}_{t}^{[1]}$ is active, while it is apparent that the constraint on the real input variable $u_{t}^{[1]}$ is far from being violated. This clearly shows that the robustness argument used to define the distributed MPC leads to a level of conservativeness in the solution of the problem which is directly proportional to the dimension of the uncertainty sets.

In Fig. 3 we compare the optimal trajectories obtained with
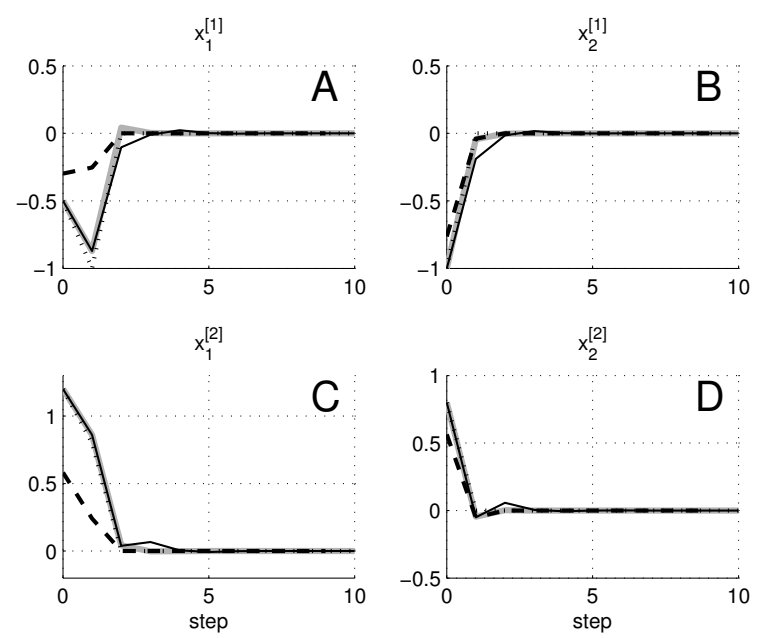

Fig. 3. Controlled state variables with dMPC (black solid lines) and with cMPC (grey solid lines), $\tilde{x}_{t}^{[i]}$ (dotted lines) and $\hat{x}_{t / t}^{[i]}$ (dashed lines). A: first element of $x_{t}^{[1]}$. B: second element of $x_{t}^{[1]}$. C: first element of $x_{t}^{[2]}$. D: second element of $x_{t}^{[2]}$.

dMPC with the ones obtained using cMPC. These results show that the performance degradation of dMPC is not significant with respect to the centralized solution.

\section{CONCLUSIONS}

In this paper we proposed a distributed MPC algorithm, whose features make it suited for practical applications. For instance, it is required limited mutual knowledge and exchange of information among neighbors, while overall convergence of the state and the possibility to handle state and control constraints are guaranteed. However, a number of significant details have still to be studied in depth to completely characterize the method (Farina and Scattolini, 2010). Among them, the need to design a decentralized auxiliary control law with the following properties: (a) it stabilizes both the local subsystems when neglecting the interconnections and the overall large scale system; (b) it has a Lyapunov function which basically corresponds to a weighted sum of local Lyapunov functions. To this regard, one can refer to milestone results (Šiljac, 1978; Sandell Jr et al., 1978; Dashkovskiy et al., 2007).

Other aspects of interest are the computation of the sets $Z_{i}$ and $E_{i}$ or the definition of suitable criteria for optimal choices of the initial reference trajectories. Note that the possibility of satisfying the fundamental constraint (8) is subject to the existence of sets $E_{i}$, implied by Assumption 3. The latter can be verified a priori (Farina and Scattolini, 2010), based on the stability properties of the decentralized auxiliary control law. The wide potentialities of the approach have still to be fully investigated. For example, since it relies on robustness concepts, it can also cope with model uncertainties e.g., on the mutual effects of the state variables of adjacent subsystems.

\section{Appendix A. PROOF OF THEOREM 1}

\section{The collective problem}

Define the collective vectors $\hat{\mathbf{x}}_{t}=\left(\hat{x}_{t}^{[1]}, \ldots, \hat{x}_{t}^{[M]}\right)$, $\tilde{\mathbf{x}}_{t}=\left(\tilde{x}_{t}^{[1]}, \ldots, \tilde{x}_{t}^{[M]}\right), \hat{\mathbf{u}}_{t}=\left(\hat{u}_{t}^{[1]}, \ldots, \hat{u}_{t}^{[M]}\right), \mathbf{w}_{t}=\left(w_{t}^{[1]}, \ldots, w_{t}^{[M]}\right)$ and $\mathbf{z}_{t}=\left(z_{t}^{[1]}, \ldots, z_{t}^{[M]}\right)$. Furthermore, define the matrices $\mathbf{A}^{*}=\operatorname{diag}\left(A_{11}, \ldots, A_{M M}\right)$ and $\tilde{\mathbf{A}}=\mathbf{A}-\mathbf{A}^{*}$. Collectively, write equations (3) and (4) as

$$
\begin{aligned}
& \mathbf{x}_{t+1}=\mathbf{A}^{*} \mathbf{x}_{t}+\mathbf{B} \mathbf{u}_{t}+\tilde{\mathbf{A}} \tilde{\mathbf{x}}_{t}+\mathbf{w}_{t} \\
& \hat{\mathbf{x}}_{t+1}=\mathbf{A}^{*} \hat{\mathbf{x}}_{t}+\mathbf{B} \hat{\mathbf{u}}_{t}+\tilde{\mathbf{A}} \tilde{\mathbf{x}}_{t}
\end{aligned}
$$

In view of (5), $\mathbf{u}_{t}=\hat{\mathbf{u}}_{t}+\mathbf{K}^{\text {aux }}\left(\mathbf{x}_{t}-\hat{\mathbf{x}}_{t}\right)$, and we collectively write (6) as

$$
\mathbf{z}_{t+1}=\left(\mathbf{A}^{*}+\mathbf{B K}^{\text {aux }}\right) \mathbf{z}_{t}+\mathbf{w}_{t}
$$

Since each $i$-MPC problem depends upon local variables (the coupling terms $\tilde{x}_{k}^{[i]}$ are fixed for $k=t, \ldots, t+N-1$ ), minimizing (9) for all $i=1, \ldots, M$ is equivalent to minimize

$$
\mathbf{V}^{N *}\left(\mathbf{x}_{t}\right)=\min _{\hat{\mathbf{x}}_{t}, \hat{\mathbf{u}}_{[t: t+N-1]}} \mathbf{V}^{N}\left(\hat{\mathbf{x}}_{t}, \hat{\mathbf{u}}_{[t: t+N-1]}\right)
$$

subject to the dynamic constraints (A.2), the static constraints

$$
\begin{gathered}
\mathbf{x}_{t}-\hat{\mathbf{x}}_{t} \in \mathbb{Z}=\prod_{i=1}^{M} Z_{i} \\
\hat{\mathbf{x}}_{k}-\tilde{\mathbf{x}}_{k} \in \mathbb{E}=\prod_{i=1}^{M} E_{i} \\
\hat{\mathbf{x}}_{k} \in \hat{\mathbb{X}} \\
\hat{\mathbf{u}}_{k} \in \hat{\mathbb{U}}
\end{gathered}
$$

for $k=t, \ldots, t+N-1$, and the terminal constraint

$$
\hat{\mathbf{x}}_{t+N} \in \hat{\mathbb{X}}^{F}
$$


Here, the cost function $\mathbf{V}^{N}$ is defined as

$$
\mathbf{V}^{N}\left(\hat{\mathbf{x}}_{t}, \hat{\mathbf{u}}_{[t: t+N-1]}\right)=\sum_{k=t}^{t+N-1} \mathbf{l}\left(\hat{\mathbf{x}}_{k}, \hat{\mathbf{u}}_{k}\right)+\mathbf{V}^{F}\left(\hat{\mathbf{x}}_{t+N}\right)
$$

We also define

$$
\mathbf{V}^{N, 0}\left(\hat{\mathbf{x}}_{t}\right)=\min _{\hat{\mathbf{u}}_{[t: t+N-1]}} \mathbf{V}^{N}\left(\hat{\mathbf{x}}_{t}, \hat{\mathbf{u}}_{[t: t+N-1]}\right)
$$

subject to the dynamic constraints (A.2) and the static constraints (A.5b)-(A.6).

\section{Feasibility}

From Definition 1, it collectively holds that

$$
\begin{aligned}
\mathbb{X}^{N}= & \left\{\mathbf{x}: \text { if } \mathbf{x}_{0}=\mathbf{x} \text { then } \exists \tilde{\mathbf{x}}_{[0: N-1]}, \hat{\mathbf{x}}_{0 / 0}, \hat{\mathbf{u}}_{[0, N-1]}\right. \\
& \text { such that (A.2), (A.5) and (A.6) are satisfied }\}
\end{aligned}
$$

and that, for each point of the feasibility set $\mathbf{x} \in \mathbb{X}^{N}$

$$
\begin{aligned}
\tilde{\mathbb{X}}_{\mathbf{x}}:= & \left\{\tilde{\mathbf{x}}_{[0: N-1]}: \text { if } \mathbf{x}_{0}=\mathbf{x} \text { then } \exists \hat{\mathbf{x}}_{0 / 0}, \hat{\mathbf{u}}_{[0, N-1]}\right. \\
& \text { such that (A.2), (A.5) and (A.6) are satisfied }\}
\end{aligned}
$$

Assume that, at instant $t, \mathbf{x}_{t} \in \mathbb{X}^{N}$ and that $\tilde{\mathbf{x}}_{[t: t+N-1]} \in \tilde{\mathbb{X}}_{\mathbf{x}_{t}}$. The optimal nominal input and state sequences obtained by minimizing the collective MPC problem are $\hat{\mathbf{u}}_{[t: t+N-1] / t}=$ $\left\{\hat{\mathbf{u}}_{t / t}, \ldots, \hat{\mathbf{u}}_{t+N-1 / t}\right\}$ and $\hat{\mathbf{x}}_{[t: t+N] / t}=\left\{\hat{\mathbf{x}}_{t / t}, \ldots, \hat{\mathbf{x}}_{t+N / t}\right\}$, respectively. Finally, recall that it is set $\tilde{\mathbf{x}}_{t+N}=\hat{\mathbf{x}}_{t+N / t}$.

Define $\hat{\mathbf{u}}_{t+N / t}^{a u x}=\mathbf{K}^{\text {aux }} \hat{\mathbf{x}}_{t+N / t}$ and compute $\hat{\mathbf{x}}_{t+N+1 / t}^{a u x}$ according to (A.2) from $\hat{\mathbf{x}}_{t+N / t}$ where $\hat{\mathbf{u}}_{t+N}=\hat{\mathbf{u}}_{t+N / t}^{\text {aux }}$. We obtain

$$
\hat{\mathbf{x}}_{t+N+1 / t}^{a u x}=\mathbf{A}^{*} \hat{\mathbf{x}}_{t+N / t}+\mathbf{B} \hat{\mathbf{u}}_{t+N / t}^{a u x}+\tilde{\mathbf{A}} \tilde{\mathbf{x}}_{t+N}
$$

since $\tilde{\mathbf{x}}_{t+N}=\hat{\mathbf{x}}_{t+N / t}$, the latter is equivalent to

$$
\hat{\mathbf{x}}_{t+N+1 / t}^{a u x}=\left(\mathbf{A}+\mathbf{B K}^{a u x}\right) \hat{\mathbf{x}}_{t+N / t}
$$

Note that, in view of constraint (A.6) and Assumption 2, $\hat{\mathbf{u}}_{t+N / t}^{a u x} \in \hat{\mathbb{U}}$ and $\hat{\mathbf{x}}_{t+N+1 / t}^{a u x} \in \hat{\mathbb{X}}^{F}$. Therefore, they satisfy constraints (A.5c), (A.5d) and (A.6). Also, according to Assumption $2,(16)$ holds.

We also define the input sequence

$$
\overline{\mathbf{u}}_{[t+1: t+N] / t}=\left\{\hat{\mathbf{u}}_{t+1 / t}, \ldots, \hat{\mathbf{u}}_{t+N-1 / t}, \hat{\mathbf{u}}_{t+N / t}^{\text {aux }}\right\}
$$

and the state sequence stemming from the initial condition $\hat{\mathbf{x}}_{t+1 / t}$ and the input sequence $\overline{\mathbf{u}}_{[t+1: t+N] / t}$ i.e.,

$$
\overline{\mathbf{x}}_{[t+1: t+N+1] / t}=\left\{\hat{\mathbf{x}}_{t+1 / t}, \ldots, \hat{\mathbf{x}}_{t+N / t}, \hat{\mathbf{x}}_{t+N+1 / t}^{\text {aux }}\right\}
$$

In view of the feasibility of the $i$-MPC problem at time $t$, we have that $\mathbf{x}_{t+1}-\hat{\mathbf{x}}_{t+1 / t} \in \mathbb{Z}$ and $\hat{\mathbf{x}}_{k / t}-\tilde{\mathbf{x}}_{k} \in \prod_{i=1}^{M} \mathbb{E}$ for all $k=t+1, \ldots, t+N-1$. Note also that $\hat{\mathbf{x}}_{t+N / t}-\tilde{\mathbf{x}}_{t+N}=0 \in \mathbb{E}$ by (15). Therefore, we can conclude that the state and the input sequences $\overline{\mathbf{x}}_{[t+1: t+N+1] / t}$ and $\overline{\mathbf{u}}_{[t+1: t+N] / t}$ are feasible at $t+1$, since constraints (A.5) and (A.6) are satisfied. This proves that $\mathbf{x}_{t} \in \mathbb{X}^{N}$ and $\tilde{\mathbf{x}}_{[t: t+N-1]} \in \tilde{\mathbb{X}}_{\mathbf{x}_{t}}$ implies that $\mathbf{x}_{t+1} \in \mathbb{X}^{N}$ and $\tilde{\mathbf{x}}_{[t+1: t+N]} \in \tilde{\mathbb{X}}_{\mathbf{x}_{t+1}}$.

\section{Convergence of the optimal cost function}

By optimality, $\mathbf{V}^{N, 0}\left(\hat{\mathbf{x}}_{t+1 / t}\right) \leq \mathbf{V}^{N}\left(\hat{\mathbf{x}}_{t+1 / t}, \overline{\mathbf{u}}_{[t+1: t+N] / t}\right)$, where

$$
\mathbf{V}^{N}\left(\hat{\mathbf{x}}_{t+1 / t}, \overline{\mathbf{u}}_{[t+1: t+N] / t}\right)=\sum_{k=t+1}^{t+N} \mathbf{l}\left(\hat{\mathbf{x}}_{k / t}, \hat{\mathbf{u}}_{k / t}\right)+\mathbf{V}^{F}\left(\hat{\mathbf{x}}_{t+N+1 / t}^{\text {aux }}\right)
$$

where it is set $\hat{\mathbf{u}}_{t+N / t}=\hat{\mathbf{u}}_{t+N / t}^{\text {aux }}$. Therefore we compute that

$$
\begin{aligned}
\mathbf{V}^{N, 0}\left(\hat{\mathbf{x}}_{t+1 / t}\right)- & \mathbf{V}^{N, 0}\left(\hat{\mathbf{x}}_{t / t}\right) \leq-\mathbf{l}\left(\hat{\mathbf{x}}_{t / t}, \hat{\mathbf{u}}_{t / t}\right)+\mathbf{l}\left(\hat{\mathbf{x}}_{t+N / t}, \hat{\mathbf{u}}_{t+N / t}^{a u x}\right)+ \\
& +\mathbf{V}^{F}\left(\hat{\mathbf{x}}_{t+N+1 / t}^{a u x}\right)-\mathbf{V}^{F}\left(\hat{\mathbf{x}}_{t+N / t}\right)
\end{aligned}
$$

In view of (16)

$$
\begin{aligned}
\mathbf{V}^{F}\left(\hat{\mathbf{x}}_{t+N+1 / t}^{\text {aux }}\right) & -\mathbf{V}^{F}\left(\hat{\mathbf{x}}_{t+N / t}\right)+\mathbf{l}\left(\hat{\mathbf{x}}_{t+N / t}, \hat{\mathbf{u}}_{t+N / t}^{\text {aux }}\right) \leq \\
& -\boldsymbol{\kappa l}\left(\hat{\mathbf{x}}_{t+N / t}, \hat{\mathbf{u}}_{t+N / t}^{\text {aux }}\right)
\end{aligned}
$$

and so, from (A.9), it follows that

$$
\mathbf{V}^{N, 0}\left(\hat{\mathbf{x}}_{t+1 / t}\right) \leq \mathbf{V}^{N, 0}\left(\hat{\mathbf{x}}_{t / t}\right)-\mathbf{l}\left(\hat{\mathbf{x}}_{t / t}, \hat{\mathbf{u}}_{t / t}\right)-\kappa \mathbf{l}\left(\hat{\mathbf{x}}_{t+N / t}, \hat{\mathbf{u}}_{t+N / t}^{a u x}\right)
$$

Recall the definition of $l_{i}$ and of matrix $R_{i}$, for all $i=1, \ldots, M$, and define $\mathbf{R}=\operatorname{diag}\left(R_{1}, \ldots, R_{M}\right)$. Then, there exists a $\mathscr{K}_{\infty}$ function $\alpha_{L}$ such that $\mathbf{l}(\mathbf{x}, \mathbf{u}) \geq \alpha_{L}(\|(\mathbf{x}, \mathbf{R u})\|)$ for all $\mathbf{x} \in \mathbb{R}^{n}, \mathbf{u} \in \mathbb{R}^{m}$. This implies that $\mathbf{l}(\mathbf{x}, \mathbf{u}) \geq \alpha_{L}(\|\mathbf{x}\|)$ for all $\mathbf{x} \in \mathbb{R}^{n}, \mathbf{u} \in \mathbb{R}^{m}$. Therefore

$$
\mathbf{V}^{N, 0}\left(\hat{\mathbf{x}}_{t+1 / t}\right) \leq \mathbf{V}^{N, 0}\left(\hat{\mathbf{x}}_{t / t}\right)-\alpha_{L}\left(\left\|\hat{\mathbf{x}}_{t / t}\right\|\right)-\kappa \alpha_{L}\left(\left\|\tilde{\mathbf{x}}_{t+N}\right\|\right)
$$

for all feasible sequences $\tilde{\mathbf{x}}_{k}, k=t, \ldots, t+N-1$.

Now we analyze the properties of the cost function $\mathbf{V}^{N *}\left(\mathbf{x}_{t}\right)$ defined in (A.4). First, note that, by definition of $\hat{\mathbf{x}}_{t / t}$, we have that $\mathbf{V}^{N *}\left(\mathbf{x}_{t}\right)=\mathbf{V}^{N, 0}\left(\hat{\mathbf{x}}_{t / t}\right)$. By optimality, we have that

$$
\mathbf{V}^{N *}\left(\mathbf{x}_{t+1}\right)=\mathbf{V}^{N, 0}\left(\hat{\mathbf{x}}_{t+1 / t+1}\right) \leq \mathbf{V}^{N, 0}\left(\hat{\mathbf{x}}_{t+1 / t}\right)
$$

Considering (A.11), we obtain that

$$
\mathbf{V}^{N *}\left(\mathbf{x}_{t+1}\right) \leq \mathbf{V}^{N *}\left(\mathbf{x}_{t}\right)-\alpha_{L}\left(\left\|\hat{\mathbf{x}}_{t / t}\right\|\right)-\kappa \alpha_{L}\left(\left\|\tilde{\mathbf{x}}_{t+N}\right\|\right)
$$

for all $\mathbf{x}_{t} \in \mathbb{X}^{N}$ and for all sequences $\tilde{\mathbf{x}}_{[t: t+N-1]} \in \tilde{\mathbf{X}}_{\mathbf{x}_{t}}$. This proves that $\left\|\hat{\mathbf{x}}_{t / t}\right\| \rightarrow 0$ and $\left\|\tilde{\mathbf{x}}_{t}\right\| \rightarrow 0$ as $t \rightarrow+\infty$.

\section{Convergence of the trajectories}

Let $\delta_{F}$ be a positive real number such that, if $\left\|\hat{\mathbf{x}}_{k}\right\|<\delta_{F}$, $\left\|\tilde{\mathbf{x}}_{k}\right\|<\delta_{F}, k=t, \ldots, t+N$ and $\left\|\hat{\mathbf{u}}_{k}\right\|<\delta_{F}, k=t, \ldots, t+N-1$, then constraints (A.5b)-(A.6) are satisfied.

Define a sequence $\overline{\mathbf{x}}_{k / t}, k=t, \ldots, t+N$, stemming from the initial condition $\overline{\mathbf{x}}_{t / t}=\hat{\mathbf{x}}_{t / t}$, whose dynamics obeys to (A.2), and where the input is $\hat{\mathbf{u}}_{k}=\overline{\mathbf{u}}_{k / t}=\mathbf{K}^{a u x} \overline{\mathbf{x}}_{k / t}$, for all $k=t, \ldots, t+$ $N-1$. Then there exists a positive real number $\delta_{x}<\delta_{F}$ such that, if $\left\|\hat{\mathbf{x}}_{t / t}\right\|<\delta_{x}$ and $\left\|\tilde{\mathbf{x}}_{k}\right\|<\delta_{x}$ for $k=t, \ldots, t+N-1$, then $\left\|\overline{\mathbf{x}}_{k / t}\right\|<\delta_{F}, k=t, \ldots, t+N$, and $\left\|\overline{\mathbf{u}}_{k / t}\right\|<\delta_{F}, k=t, \ldots, t+N-$ 1. In fact, denoting $\mathbf{F}=\mathbf{A}^{*}+\mathbf{B K}^{a u x}$, from (A.2), for $i \geq 1$

$$
\overline{\mathbf{x}}_{t+i / t}=\mathbf{F}^{i} \hat{\mathbf{x}}_{t / t}+\sum_{j=0}^{i-1} \mathbf{F}^{j} \tilde{\mathbf{A}} \tilde{\mathbf{x}}_{t+i-j-1}
$$

and $\left\|\overline{\mathbf{x}}_{t / t}\right\|=\left\|\hat{\mathbf{x}}_{t / t}\right\|<\delta_{x}<\delta_{F},\left\|\overline{\mathbf{x}}_{t+i / t}\right\|<\max _{i=1, \ldots, N} \| \mathbf{F}^{i}+$ $\sum_{j=0}^{i-1} \mathbf{F}^{j} \tilde{\mathbf{A}} \| \delta_{x}$ and $\left\|\overline{\mathbf{u}}_{k / t}\right\| \leq\left\|\mathbf{K}^{\text {aux }}\right\|\left\|\overline{\mathbf{x}}_{k / t}\right\|$.

Therefore, for a suitable $\delta_{x}$, if $\left\|\hat{\mathbf{x}}_{t / t}\right\|<\delta_{x}$ and $\left\|\tilde{\mathbf{x}}_{k}\right\|<\delta_{x}$, $k=t, \ldots, t+N-1$, then the trajectories $\overline{\mathbf{x}}_{k / t}, k=t, \ldots, t+$ $N$ and $\overline{\mathbf{u}}_{k / t}, k=t, \ldots, t+N-1$ are feasible (since also $\hat{\mathbf{x}}_{t / t}$ satisfies (A.5a) for the feasibility of the $i$-MPC problem at time $t$ ).

Since $\left\|\hat{\mathbf{x}}_{t / t}\right\| \rightarrow 0$ and $\left\|\tilde{\mathbf{x}}_{t}\right\| \rightarrow 0$ as $t \rightarrow+\infty$, there exists $\bar{t}>0$ such that $\left\|\hat{\mathbf{x}}_{t / t}\right\|<\delta_{x}$ and $\left\|\tilde{\mathbf{x}}_{t}\right\|<\delta_{x}$ for all $t \geq \bar{t}$, which makes the trajectories $\overline{\mathbf{x}}_{k / t}, k=t, \ldots, t+N$, and $\overline{\mathbf{u}}_{k / t}, k=t, \ldots, t+N-$ 1 , feasible for all $t \geq \bar{t}$. By optimality, if $t \geq \bar{t}$

$$
\mathbf{V}^{N *}\left(\mathbf{x}_{t}\right)=\mathbf{V}^{N, 0}\left(\hat{\mathbf{x}}_{t / t}\right) \leq \sum_{k=t}^{t+N-1} \mathbf{l}\left(\overline{\mathbf{x}}_{k / t}, \overline{\mathbf{u}}_{k / t}\right)+\mathbf{V}^{F}\left(\overline{\mathbf{x}}_{t+N / t}\right)
$$

Recall (16). Since $\mathbf{V}^{F} \geq 0$ by definition, one has that $\mathbf{l}\left(\overline{\mathbf{x}}_{k / t}, \overline{\mathbf{u}}_{k / t}\right) \leq$ $\frac{1}{1+\kappa} \mathbf{V}^{F}\left(\overline{\mathbf{x}}_{k / t}\right) \leq \mathbf{V}^{F}\left(\overline{\mathbf{x}}_{k / t}\right)$ and, from (A.14) 


$$
\mathbf{V}^{N *}\left(\mathbf{x}_{t}\right) \leq \sum_{k=t}^{t+N} \mathbf{V}^{F}\left(\overline{\mathbf{x}}_{k / t}\right)
$$

From (A.13) and (A.15), we obtain that, for all $t \geq \bar{t}$, there exists a $\mathscr{K}_{\infty}$ function $\beta$ such that

$$
\mathbf{V}^{N *}\left(\mathbf{x}_{t}\right) \leq \beta\left(\left\|\left(\hat{\mathbf{x}}_{t / t}, \tilde{\mathbf{x}}_{[t: t+N-1]}\right)\right\|\right)
$$

For this it follows that $\mathbf{V}^{N *}\left(\mathbf{x}_{t}\right) \rightarrow 0$ as $t \rightarrow+\infty$.

Recall that $\hat{\mathbf{x}}_{k / t}$ is generated according to (A.2), stemming from the optimal initial condition $\hat{\mathbf{x}}_{t / t}$ and inputs $\hat{\mathbf{u}}_{k / t}$. One can write the solution to (A.2) as $\hat{\mathbf{x}}_{t+i / t}=\mathbf{v}_{t+i / t}+\mathscr{B}_{i} \mathbf{U}_{t}$, where

$$
\begin{aligned}
\mathbf{v}_{t+i / t} & =\left(\mathbf{A}^{*}\right)^{i} \hat{\mathbf{x}}_{t / t}+\sum_{j=0}^{i-1}\left(\mathbf{A}^{*}\right)^{j} \tilde{\mathbf{A}} \tilde{\mathbf{x}}_{t+i-j-1}, \\
\mathscr{B}_{i} & =\left[\begin{array}{llllll}
\left(\mathbf{A}^{*}\right)^{i-1} \mathbf{B} & \ldots & \mathbf{B} & 0 & \ldots & 0
\end{array}\right]
\end{aligned}
$$

if $i=1, \ldots, N, \mathbf{U}_{t}=\left(\hat{\mathbf{u}}_{t / t}, \ldots, \hat{\mathbf{u}}_{t+N-1 / t}\right)$. Note that, since $\left\|\hat{\mathbf{x}}_{t / t}\right\| \rightarrow 0$ and $\left\|\tilde{\mathbf{x}}_{t}\right\| \rightarrow 0$ as $t \rightarrow+\infty$, also $\left\|\mathbf{v}_{k / t}\right\| \rightarrow 0$ as $t \rightarrow+\infty$ for all $k=t+1, \ldots, t+N$. We also denote $\mathbf{v}_{t / t}=\hat{\mathbf{x}}_{t / t}$ and $\mathscr{B}_{0}=0_{n \times N m}$.

Now, consider again the function $\mathbf{V}^{N *}\left(\mathbf{x}_{t}\right)$ :

$\mathbf{V}^{N *}\left(\mathbf{x}_{t}\right)=\sum_{k=t}^{t+N-1} \mathbf{l}\left(\mathbf{v}_{k / t}+\mathscr{B}_{k-t} \mathbf{U}_{t}, \hat{\mathbf{u}}_{k / t}\right)+\mathbf{V}^{F}\left(\mathbf{v}_{t+N / t}+\mathscr{B}_{N} \mathbf{U}_{t}\right)$

From the definition of $l_{i}$ it follows that $\mathbf{l}\left(\mathbf{x}_{k}, \mathbf{u}_{k}\right) \geq \alpha_{L}\left(\left\|\left(\mathbf{x}_{k}, \mathbf{R} \mathbf{u}_{k}\right)\right\|\right)$, and so

$$
\begin{aligned}
0 \leq & \sum_{k=t}^{t+N-1} \alpha_{L}\left(\left\|\left(\mathbf{v}_{k / t}+\mathscr{B}_{k-t} \mathbf{U}_{t}, \mathbf{R} \hat{\mathbf{u}}_{k / t}\right)\right\|\right) \\
& +\mathbf{V}^{F}\left(\mathbf{v}_{t+N / t}+\mathscr{B}_{N} \mathbf{U}_{t}\right) \leq \mathbf{V}^{N *}\left(\mathbf{x}_{t}\right)
\end{aligned}
$$

Since it is proved that $\mathbf{V}^{N *}\left(\mathbf{x}_{t}\right) \rightarrow 0$ as $t \rightarrow+\infty$, it follows that, for all $k=t, \ldots, t+N-1$

$$
\alpha_{L}\left(\left\|\left(\mathbf{v}_{k / t}+\mathscr{B}_{k-t} \mathbf{U}_{t}, \mathbf{R} \hat{\mathbf{u}}_{k / t}\right)\right\|\right) \rightarrow 0
$$

and $\mathbf{V}^{F}\left(\mathbf{v}_{t+N / t}+\mathscr{B}_{N} \mathbf{U}_{t}\right) \rightarrow 0$ as $t \rightarrow+\infty$. This implies that:

$$
\mathbb{B} \mathbf{U}_{t}+\mathbf{V}_{t} \rightarrow 0
$$

as $t \rightarrow \infty$, where

$$
\mathbb{B}=\left[\begin{array}{c}
\overline{\mathscr{B}} \\
\operatorname{diag}(\mathbf{R}, \ldots, \mathbf{R})
\end{array}\right], \overline{\mathscr{B}}=\left[\begin{array}{lll}
\mathscr{B}_{0}^{T} & \ldots & \mathscr{B}_{N}^{T}
\end{array}\right]^{T}
$$

and $\mathbf{V}_{t}=\left(v_{t / t}, \ldots, v_{t+N / t}, 0, \ldots, 0\right)$. It is readily seen that, in view of the triangular structure of $\overline{\mathscr{B}}$ and since, by definition of $R_{i}, i=1, \ldots, M, \operatorname{rank}\left(\left[\mathbf{B}^{T} \mathbf{R}^{T}\right]^{T}\right)=m$ then $\operatorname{rank}(\mathbb{B})=N m$. Since $\mathbf{V}_{t} \rightarrow 0$ as $t \rightarrow+\infty$, from (A.18) it follows that $\mathbf{U}_{t} \rightarrow 0$ as $t \rightarrow+\infty$. Therefore $\hat{\mathbf{u}}_{t / t} \rightarrow 0$ as $t \rightarrow+\infty$.

Finally, recall that the state $\mathbf{x}_{t}$ evolves according to the equation

$$
\begin{aligned}
\mathbf{x}_{t+1} & =\mathbf{A} \mathbf{x}_{t}+\mathbf{B}\left[\hat{\mathbf{u}}_{t / t}+\mathbf{K}^{\text {aux }}\left(\mathbf{x}_{t}-\hat{\mathbf{x}}_{t / t}\right)\right] \\
& =\left(\mathbf{A}+\mathbf{B K}^{\operatorname{aux}}\right) \mathbf{x}_{t}+\mathbf{B}\left(\hat{\mathbf{u}}_{t / t}-\mathbf{K}^{\text {aux }} \hat{\mathbf{x}}_{t / t}\right)
\end{aligned}
$$

By asymptotic convergence to zero of the nominal state and input signals $\hat{\mathbf{x}}_{t / t}$ and $\hat{\mathbf{u}}_{t / t}$ respectively, we obtain that $\mathbf{B}\left(\hat{\mathbf{u}}_{t / t}-\mathbf{K}^{\text {aux }} \hat{\mathbf{x}}_{t / t}\right)$ is an asymptotically vanishing term. Since also $\left(\mathbf{A}+\mathbf{B K}^{a u x}\right)$ is Schur by Assumption 2, we obtain that $\mathbf{x}_{t} \rightarrow 0$ as $t \rightarrow+\infty$. This concludes the proof of Theorem 1 .

\section{REFERENCES}

Barcelli, D. and Bemporad, A. (2009). Decentralized model predictive control of dynamically-coupled linear systems:
Tracking under packet loss. Proc. 1st IFAC Workshop on Estimation and Control of Networked Systems, Venice, Italy.

Dashkovskiy, S., Rüffer, B.S., and Wirth, F. (2007). An ISS small gain theorem for ISS general networks. Mathematics of Control, Signals, and Systems, 19(2), 93-122.

Dunbar, W. (2007). Distributed receding horizon control of dynamically coupled nonlinear systems. IEEE Trans. on Automatic Control, 52, 1249-1263.

Dunbar, W. and Murray, R. (2006). Distributed receding horizon control for multi-vehicle formation stabilization. Automatica, 42, 549-558.

Farina, M. and Scattolini, R. (2010). Distributed predictive control: a non-cooperative algorithm with neighbor-to-neighbor communication. Submitted.

Ferrari-Trecate, G., Galbusera, L., Marciandi, M., and Scattolini, R. (2009). Model predictive control schemes for consensus in multi-agent systems with single- and doubleintegrator dynamics. IEEE Trans. on Automatic Control, 54(11), $2560-2572$.

Liu, J., Chen, X., Muñoz de la Peña, D., and Christofides, P. (2010). Sequential and iterative architectures for distributed model predictive control of nonlinear process systems. part I: theory. Proceedings of American Control Conference, 3148 -3155 .

Liu, J., Muñoz de la Peña, D., and Christofides, P. (2009). Distributed model predictive control of nonlinear process systems. AIChE J., 55(9), 1171-1184.

Mayne, D., Seron, M., and Rakovic, S.V. (2005). Robust model predictive control of constrained linear systems with bounded disturbances. Automatica, 41, 219-224.

Negenborn, R., van Overloop, P.J., and De Schutter, B. (2009). Coordinated distributed model predictive reach control of irrigation canals. Proceedings of the European Control Conference, 1420-1425.

Raimondo, D.M., Magni, L., and Scattolini, R. (2006). Decentralized MPC of nonlinear systems: an input-to-state stability approach. International Journal of Robust and Nonlinear control, 17, $1651-1667$.

Raković, S.V., Kerrigan, E.C., Kouramas, K.I., and Mayne, D. (2005). Invariant approximations of the minimal robust positively invariant set. IEEE Trans. Aut. Cont, 50(3), $406-$ 410.

Richards, A. and How, J. (2007). Robust distributed model predictive control. International Journal of Control, 80(9), 1517-1531.

Sandell Jr, N., Varaiya, P., Athans, M., and Safonov, M. (1978). Survey of decentralized control methods for large scale systems. IEEE Trans. on Automatic Control, 23(2), 108 - 128.

Scattolini, R. (2009). Architectures for distributed and hierarchical model predictive control. Journal of Process Control, $19,723-731$.

Stewart, B.T., Venkat, A.N., Rawlings, J.B., Wright, S.J., and Pannocchia, G. (2010). Cooperative distributed model predictive control. System \& Control Letters, 59, 460-469.

Trodden, P. and Richards, A. (2010). Distributed model predictive control of linear systems with persistent disturbance. International Journal of Control, 83(8), 1653 - 1663.

Venkat, A.N., Hiskens, I., Rawlings, J.B., and Wright, S.J. (2008). Distributed MPC strategies with application to power system automatic generation control. IEEE Trans. Automatic Control, 16(6), 1192 - 1206.

Šiljac, D.D. (1978). Large-scale dynamic systems. Stability and structure. North Holland. 